\title{
Dark Energy is Stellar Nuclear Fusion Replicated in a Mirrored Universe
}

\author{
Leong Ying*
}

Princeton Gamma-Tech Instruments, 303C College Road East, Princeton, NJ 08540, USA

\begin{abstract}
From a Twin Universe perspective, it is proposed that stellar nuclear fusion can account for the negative-energy pressure (Dark Energy) that drives our present-day accelerated cosmic expansion. In the mirror twinned universe all processes are duplicated but with reverse negative polarity. Both the Positive and Negative Universes exist on the opposing surfaces of a topological two-dimensional membrane and therefore shares the same experience of a stretching membrane.
\end{abstract}

Keywords: Dark Energy, polarity, cosmic expansion, Twin Universe.

\section{INTRODUCTION}

Using thermodynamic conservation principles the cosmos existing as a pair of identical anti-parallel universes has been proposed [1]. Parameterization of negative quantities can be formulated in terms of Santilli's isodual theory of antimatter [2]. Sakharov [3] proposed the breaking of charge-parity $(C P)$ symmetry led to the baryonic imbalance that created a surplus of matter in our present-day universe. Petit [4] expanded on this cosmological model whereby a twin universe with reverse arrow of time $(T)$ populated mainly by antimatter would maintain global un-violated $C P T$-symmetry between the combined universes. The new proposed model postulates that twin universes exist in an identical state of duality, whereby all contents and processes of each universe have equal magnitude but opposite polarity, including energy-mass that in the anti-parallel universe will have negative quantities producing the necessary gravitational repulsion to drive the cosmic expansion. Since both universes reside on the topological surfaces of a shared membrane, the effective stretching of this common cosmic membrane will be observed as expansion in our side of the universe.

The Wilkinson Microwave Anisotropy Probe [5] has determined the Hubble's constant $(\mathrm{H})$ at $71 \mathrm{~km} \cdot \mathrm{s}^{-1} \cdot \mathrm{Mpc}^{-1}$. Hubble's law [6] is considered the first observational basis for an expanding universe and supporting evidence of the Big Bang model. Dark Energy is the unknown constituent that propels the present accelerating state of our cosmos, and the equally mysterious Dark Matter conjured up to explain the highrotational velocities of galaxies. The rest of this article proposes that normal nuclear fusion in stars can account for these unknown cosmic mechanisms without the need for such dark fluids.

\section{STELLAR NUCLEAR FUSION}

Stellar nucleosynthesis is the process of nuclear reactions taking place in stars to build heavier elements. The net mass

*Address correspondence to this author at Princeton Gamma-Tech Instruments, 303C College Road East, Princeton, NJ 08540, USA; Tel: 609-9247310 x-270; Fax: 609-924-1729;

E-mails: leong.ying@pgt.com; leong@klystar.com of fused nuclei is smaller than the sum of the components, with the loss mass released as electromagnetic energy according to Einstein's famous mass-energy equivalence relationship:

$E=m c^{2}$

Newton's law of universal gravity states that the force between two point masses $\left(\mathrm{m}_{1}, \mathrm{~m}_{2}\right)$ a distance $\mathrm{r}$ apart is given by the following equation:

$F=G \frac{m_{1} m_{2}}{r^{2}}$

If we assume that the masses are of equal magnitude $\mathrm{m}=\mathrm{m}_{1}=\mathrm{m}_{2}$, and the area mass density condensing on the twodimensional membrane $\rho_{\mathrm{m}}=\mathrm{m} / \pi \mathrm{r}^{2}$, then the gravitational force of acceleration produced by one point mass on the other is given by:

$a=\pi G \rho_{m}$

\section{ENERGY PRODUCTION IN STARS}

The observable universe is composed of $70 \%$ hydrogen, and the proton-proton ( $p-p)$ chain reaction is the predominant thermonuclear fusion process that converts hydrogen nuclei into helium in stars with masses up to that of the Sun.

$4^{1} \mathrm{H} \rightarrow{ }^{4} \mathrm{He}+2 \mathrm{e}^{+}+2 \mathrm{v}_{\mathrm{e}}$

Along with the formation of a pair of positrons and neutrinos, $26.7 \mathrm{MeV}$ of energy is released, equivalent to a mass of $4.8 \times 10^{-29} \mathrm{~kg}$.

For more massive stars, another reaction process is also important that of the carbon-nitrogen-oxygen $(\mathrm{CNO})$ cycle. In the main CNO-I reaction the carbon can be considered a catalyst in converting hydrogen into helium with the carbon being reformed at the completion of the following cycle:

${ }^{12} \mathrm{C} \rightarrow{ }^{13} \mathrm{~N} \rightarrow{ }^{13} \mathrm{C} \rightarrow{ }^{14} \mathrm{~N} \rightarrow{ }^{15} \mathrm{O} \rightarrow{ }^{15} \mathrm{~N} \rightarrow{ }^{12} \mathrm{C}$ 
Table 1. Cosmological History of Expanding Twin Universes

\begin{tabular}{|c|c|c|}
\hline Timeline & Content of Universes & Cosmic Expansion Rates \\
\hline $\begin{array}{l}\text { Planck epoch } \\
0 \text { to } 10^{-43} \mathrm{~s}\end{array}$ & $\begin{array}{l}\text { Pure energy in positive universe. } \\
\text { Pure mass in negative universe. }\end{array}$ & $\begin{array}{l}\text { Point source grows to Planck size }\left(1=4 \times 10^{-35} \mathrm{~m}\right) \text { at infi- } \\
\text { nite rate. }\end{array}$ \\
\hline $\begin{array}{l}\text { Inflation } \\
10^{-43} \text { to } 10^{-37} \mathrm{~s}\end{array}$ & $\begin{array}{l}\text { Energy in positive universe. } \\
\text { Formation of divided matter at exponential rate in nega- } \\
\text { tive universe. }\end{array}$ & $\begin{array}{l}\mathrm{m}_{0} \sim 10^{60} \mathrm{~kg} \\
\mathrm{r}_{0} \sim 10^{-34} \mathrm{~m} \\
\mathrm{U}_{0} \sim 10^{109} \mathrm{~s}^{-1}\left(\sim 10^{129} \mathrm{~km} \cdot \mathrm{s}^{-1} \cdot \mathrm{Mpc}^{-1}\right)\end{array}$ \\
\hline Matter condensation & $\begin{array}{l}\text { Formation of baryons and leptons in positive universe. } \\
\text { Energy waves dilute matter in negative universe. }\end{array}$ & $\begin{array}{l}\mathrm{m}_{+} \text {increases, } \mathrm{m} \text {. decreases } \\
\mathrm{r} \text { maintain constancy } \\
\mathrm{U}_{\mathrm{r}} \text { decreases }\end{array}$ \\
\hline Star formation & $\begin{array}{l}\text { Present-day structure of observed positive universe. } \\
\text { Fusion energy drives expansion in negative universe. }\end{array}$ & $\begin{array}{l}\mathrm{m} \sim 10^{-29} \mathrm{~kg} \\
\mathrm{r} \sim 10^{-15} \mathrm{~m} \\
\mathrm{U}_{\mathrm{r}} \sim 10^{-18} \mathrm{~s}^{-1}\left(\sim 100 \mathrm{~km} \cdot \mathrm{s}^{-1} \cdot \mathrm{Mpc}^{-1}\right)\end{array}$ \\
\hline Extinction & $\begin{array}{l}\text { Steady state in positive universe. } \\
\text { Steady state in negative universe. }\end{array}$ & $\begin{array}{l}\mathrm{m}_{+} \text {and } \mathrm{m} \text {. constant } \\
\mathrm{r} \text { constant } \\
\mathrm{U}_{\mathrm{r}}=0\end{array}$ \\
\hline
\end{tabular}

As with the p-p cycle, the total released of energy is $26.7 \mathrm{MeV}$ due to the mass difference between the fusion of the hydrogen parents to form the helium daughter.

\section{STRETCHED UNIVERSAL MEMBRANE}

If we assume that the cosmos is uniformly distributed with point-like stars, the vast empty interstellar space would produce minimal gravitation contraction on the membrane upon which our positive universe resides. If we further assume that an identical negative universe resides on the opposite side of the same membrane, and all quantities and processes are duplicated but in opposite polarity. The stars in the positive universe would undergo the standard nuclear fusion processes that release large amount of electromagnetic waves into the empty space. Consider the same processes on the reverse side of the membrane, whereby the equivalent release of energy condenses out as point masses with negative energy and hence repulsive gravity.

For simplicity of computation, we will assume that the release of solar energy from the various chain reactions condense out as two equal point masses that occupy the volume of the fused helium nuclei $\sim 2 \times 10^{-15} \mathrm{~m}$. With a gravitational constant value of $\mathrm{G}=6.67428 \times 10^{-11} \mathrm{~m}^{3} \cdot \mathrm{kg}^{-1} \cdot \mathrm{s}^{-2}$, the gravitation repulsive acceleration is approximately $1.6 \times 10^{-9} \mathrm{~m} \cdot \mathrm{s}^{-2}$. This repulsive force of gravity moves at the speed of light $\left(c=299,792,458 \mathrm{~m} \cdot \mathrm{s}^{-1}\right)$, so the stretched membrane will expand at a rate of:

$U_{r}=\frac{\pi G \rho_{m}}{c}$

Inputting the model values, the estimated cosmic expansion rate $\mathrm{U}_{\mathrm{r}} \sim 5 \times 10^{-18} \mathrm{~s}^{-1}$. Multiplying this quantum scale of repulsive expansion over an astronomical distance of a Mega-parsec $\left(\mathrm{Mpc}=3.0857 \times 10^{22} \mathrm{~m}\right)$ gives a cosmological expansion rate of $160 \mathrm{~km} . \mathrm{s}^{-1} \cdot \mathrm{Mpc}^{-1}$. Even with this simple model the computed value for the rate of expansion is in reasonable agreement with the present-day measured Hubble constant. Alternatively by interpreting with this simple model, the current Hubble constant of $\sim 70 \mathrm{~km} . \mathrm{s}^{-1} . \mathrm{Mpc}^{-1}$ would equate to an average simulated helium fusion energy release of $11.7 \mathrm{MeV}$ or equivalent mass density $\rho_{\mathrm{m}} \sim 16 \mathrm{~kg} . \mathrm{m}^{-2}$.

\section{COSMIC HISTORY}

Using the basic expansion model defined, the history of the twin-universe cosmos can be predicted in Table 1:

\section{SUMMARY}

The Twin Universe model predicts that the complete cosmos exists as a ten-dimensional entity with two identical but anti-parallel four-dimensional space-time (energyentropy) universes residing on the opposing surfaces of a two-dimensional common membrane. Quantities and processes on both sides of the universes are duplicated but of reverse polarity. In the Positive Universe the fusion reactions within stars release vast quantities of energy into the expanse of space as electromagnetic waves. In the reverse Negative Universe the same fusion energy condenses as point masses with negative quantity (repulsive gravity) that stretches out the common membrane producing the observable accelerating expansion of the entire cosmos. Table 2 summarizes the key parameters of both mirrored universes.

Table 2. Cosmic Processes on Both Sides of the Twin Universes

\begin{tabular}{|l|l|}
\hline \multicolumn{1}{|c|}{ Positive Universe } & \multicolumn{1}{c|}{ Negative Universe } \\
\hline \hline Fusion generates energy waves & Fusion generates matter particles \\
\hline Attractive gravity & Repulsive gravity \\
\hline $\begin{array}{l}\text { Measured Hubble's constant } \\
\mathrm{H} \sim 71 \mathrm{~km} . \mathrm{s}^{-1} \cdot \mathrm{Mpc}^{-1}\end{array}$ & $\begin{array}{l}\text { Computed Universal expansion rate } \\
\mathrm{U}_{\mathrm{r}} \sim 160 \mathrm{~km} \cdot \mathrm{s}^{-1} \cdot \mathrm{Mpc}^{-1}\end{array}$ \\
\hline
\end{tabular}

The model further predicts that Dark Matter and Dark Energy constitute half of the missing observable energy- 
mass in the cosmos. Present experimental measurements estimate the percentage of Dark Matter at 23\% and Dark Energy at $73 \%$. However, there are proponents that claim both are the same component of Dark Fluid [7], and hence if the differing effects are producing a double-counting of the same unobserved material, then the actual percentage may indeed be $50 \%$.

\section{REFERENCES}

[1] Ying L. Nuclear fusion drives present-day accelerated cosmic expansion, Hadronic J 2009; 32: 573-88.
[2] Santilli RM. Isodual theory of antimatter. Dordrecht: Springer 2006. ISBN: 1402045174

[3] Sakharov AD. Violation of CP summetry, C-asymmetry and baryon asymmetry of the universe. JETP Lett 1967; 5: 24-7.

[4] Petit JP. Enantiomorphic universes with opposite time arrows. Comptes rendus de l'Academie des Sciences 1977; 263: 1315-8.

[5] Bennett CL, Bay M, Halpern M, et al. The microwave anisotropy probe mission. Astrophys J 2003; 583: 1-23.

[6] Hubble E. A relation between distance and radial velocity among extra-galactic nebulae. Proc Natl Acad Sci USA 1929; 15: 168-73.

[7] Zhao HS. An ecological approach to problems of dark energy, dark matter, MOND and neutrinos. J Phys Conf Ser 2008; 140: 1-12.

() Leong Ying; Licensee Bentham Open.

This is an open access article licensed under the terms of the Creative Commons Attribution Non-Commercial License (http://creativecommons.org/licenses/by-nc/3.0/) which permits unrestricted, non-commercial use, distribution and reproduction in any medium, provided the work is properly cited. 